\title{
Investment Horizon Effects
}

\author{
Javier Gil-Bazo*
}

\begin{abstract}
Boudry and Gray (2003) have documented that the optimal buy and hold demand for Australian stocks is not necessarily increasing in the investment horizon when returns are predictable. Such finding is in contrast with Barberis (2000) who shows that positive monotonic horizon effects predominate for US stocks. Using a closed form approx imation to the asset allocation problem, this paper relates the return dynamics to the investor's portfolio choice for different investment horizons. In the special case of a single risky asset, it is shown that return predictability under stationarity may induce both positive and negative horizon effects in the optimal allocation to the risky asset. The paper extends previous empirical results by solving for the optimal portfolio when two risky assets with predictable returns are available for investment.
\end{abstract}

Keywords: portfolio choice, return predictability, investment horizon effects

\section{INTRODUCTION}

Time diversification is based on the idea that above average risky asset returns tend to be offset by below average returns over long horizons if returns are not perfectly serially correlated. As a consequence, the variance of the annualized return diminishes with the number of years. Advocates of time diversification conclude that investors with longer horizons should hold a higher proportion of their portfolio in the risky asset. Such conclusion, however, has been subject to two main criticisms. ${ }^{1}$ First, classic results by Samuelson (1969) and Merton (1969 and 1971) demonstrate that if the investor is free to rebalance his portfolio each period, he will choose a constant allocation to the risky asset regardless of the investment horizon, provided that he either has log utility preferences, or he has constant relative risk aversion and returns are independently and identically distributed (i.i.d.). Second, the variance of total return on the risky asset not only does not decrease, but actually increases with the investor's horizon for i.i.d. single period returns. This is because although the probability of loss decreases with the investment horizon,

He is grateful for comments from an anonymous referee and Peter F. Pope (the editor), as well as from Gonzalo Rubio, Roberto Blanco and participants at the SIRIF Conference on Dynamic Portfolio Strategies and IX Foro de Finanzas. Financial support from Spanish Ministry of Science and Technology, grant SEJ2004 01688/ECON, is also acknowledged.

* The author is from the Universidad Carlos III de Madrid. (Paper received September 2004, revised version accepted June 2005.)

Address for correspondence: Javier Gil Bazo, Universidad Carlos III de Madrid, calle Madrid 126, 28903 Getafe, Madrid, Spain.

e mail: javier.gil.bazo@uc3m.es

1 For recent reviews of the time diversification literature, see, for instance, Kritzman (1994), Bodie (1995), Connelly (1996), or Kritzman and Rich (1998). 
the magnitude of the potential loss increases with time. ${ }^{2}$ Long term investments in stocks are therefore not safer than short term investments for i.i.d. returns.

If returns are mean reverting, however, then the variance of total return increases, but it does so at a lower rate than in the i.i.d. case. This implies that stocks can be relatively less risky in the long run, which may lead investors to favor risky assets over long horizons. Kritzman and Rich (1998) address this issue in a simple and clarifying context. In particular, they study the problem of an investor who must choose between investing all his wealth in a safe asset and investing it in a risky asset. Under the assumption that the stock price process can be represented by a binomial tree with mean reverting returns, they show numerically that a power utility investor with a constant relative risk aversion coefficient of two, will be indifferent between the risky asset and the safe asset in the short run, but will prefer the risky asset for a long term investment. On the other hand, a longer investment horizon will lead the investor to choose the safe asset if the return distribution is mean averting, even if he was indifferent over a short horizon. Finally, for i.i.d. returns, the investor obtains the same expected utility from investing in the risky asset and the safe asset regardless of the investment horizon.

Barberis (2000) extends the analysis to a more complex setting. More specifically, he studies the effect of the investment horizon on the optimal portfolio choice of a power utility investor assuming a lognormal return generating model with a first order autoregressive predictive variable ${ }^{3}$ driving the time varying conditional mean of log excess returns. Barberis (2000) also allows for a continuum of portfolio alloca tions between the risk free and the risky asset. Using a numerical technique, Barberis (2000) shows that the optimal buy and hold investment in stocks is increasing in the investment horizon, independently of the initial value of the predictor. Barberis (2000) argues that predictability induces mean reversion in stock expected returns, which in turn makes the variance of cumulative returns grow less than linearly as the horizon increases. As a result, a risk averse long term investor should allocate more to stocks than a short term investor, consistently with Kritzman and Rich (1998).

The expected utility analysis of long term portfolio choice under mean reversion, therefore, seems to have resurrected the idea of time diversification. This view, however, has been challenged by the empirical findings of Boudry and Gray (2003). Extending Barberis's (2000) approach to multiple predictors and Australian data, they find that some initial values of the predictors can cause the investor to allocate less to the risky asset as the investment horizon increases. Another source of discrepancy is the fact that optimal allocations are insensitive to the investment horizon in the long term. Finally, the effect of the investment horizon is not necessarily monotonic, as opposed to the US case.

Our limited understanding of investment horizon effects is perhaps explained by the lack of a closed form solution to the static portfolio choice problem in the power utility case. In this paper, we shed light on the issue by using an analytical approxi mation to the investor's objective function that permits us to relate the return dynamics to the investor's optimal asset allocation for different horizons. The approximation has the traditional mean variance form, except that risky asset returns

2 Time diversification is just an example of Samuelson's (1963) fallacy of large numbers, which he summarized with the following sentence (p. 54): 'it is not so much by adding new risks as by subdividing risks among more people that insurance companies reduce the risk of each.'

3 For evidence on return predictability, see, for instance, Campbell (1987), Campbell and Shiller (1988a and 1988b), Fama (1984 and 1990), or Fama and French (1988 and 1989). 
are replaced by log returns and expected returns must be appropriately adjusted. In a portfolio choice model with two risky assets and multiple predictors, we then investigate how model parameters influence the evolution of the risk return trade off with the investment horizon. Interestingly, under the stationarity assumption, all investment horizon effects disappear in the long horizon limit. In the special case of a single risky asset, we show that under return predictability and negative serial correlation in asset returns, the horizon effect on the optimal demand for the risky asset can be either positive or negative. Two reasons explain this conclusion. First, changes in the condi tional variance of cumulative log returns are not necessarily monotonic in the invest ment horizon: even if returns are negatively serially correlated, the asset's riskiness may grow less than linearly at short horizons and more than linearly at long horizons. Second, predictability implies that conditional expected cumulative returns may also grow less than linearly as the investment horizon grows to infinity. Intuitively, when the predictive variable is stationary, it tends to revert to its mean, so if it has a positive (negative) effect on expected returns and the initial value is above (below) its long run mean, then future expected returns will tend to decrease. This decay in the mean may offset the positive horizon effect induced by lower relative risk. Empirical results for the US are therefore explained by a very low speed of mean reversion.

We illustrate the analysis empirically by computing the optimal demand of a buy and hold investor for long term US Government bonds and stocks. While investment horizon effects are always positive when a stock portfolio is the only risky investable asset, the optimal demand for long term bonds displays both positive and negative horizon effects depending on the initial value of the predictive variable and, in any case, disappear as the investment horizon grows to infinity. When both risky assets are simultaneously available for investment, we find that the investment horizon affects not only the optimal investment in the risky asset portfolio, but also the composition of the risky portfolio itself. Moreover, investment horizon effects have different signs for different initial values of the predictors. Finally, the paper shows how these results are changed when estimation risk is incorporated explicitly in the investor's problem.

To summarize, the paper's contribution to the literature is two fold. First, it provides a theoretical analysis that relates the evolution of the risky asset return distribution over time to the optimal portfolio of a utility maximizing investor. This analysis enables us to reconcile the empirical evidence on investment horizon effects under return predictability previously documented in the literature. Second, it extends Barberis's (2000) and Boudry and Gray's (2003) approach to the case when two risky assets are available for investment.

The rest of the paper is divided into the following sections: Section 2 presents the theoretical framework of the paper; Section 3 documents the empirical results; and finally, Section 4 concludes.

\section{PORTFOLIO CHOICE UNDER RETURN PREDICTABILITY}

In this section, we start by presenting the multiperiod portfolio problem of an investor with power utility who does not rebalance his portfolio. Throughout the paper, we follow Barberis (2000), but we consider two natural extensions of the original setting. First, we allow for multiple regressors in the predictive equation as in Boudry and Gray (2003), and, second, we include the possibility to invest in multiple risky assets. In the second part of the section, we investigate investment horizon effects for the special cases of two and one risky assets. 
(i) The Investor's Problem

Consider the problem at time $T$ of an investor who derives utility from his wealth at time $T+\hat{T}$. The investor does not rebalance his portfolio and can invest in $N+1$ different assets: the one month Treasury Bill with constant interest rate, $r_{f}$; and $N$ risky assets. The continuously compounded return in excess of the riskless interest rate on the $i$ th risky asset between periods $t \quad 1$ and $t$ is denoted by $r_{i t}$.

If initial wealth equals one and $\boldsymbol{\omega}^{\prime}=\left[\begin{array}{llll}\omega_{1} & \omega_{2} \ldots \omega_{N}\end{array}\right]$ contains the fraction of the investor's initial wealth allocated to each risky asset, then the investor's terminal wealth is given by:

$$
W_{T+\hat{T}}=\left(\begin{array}{ll}
1 & \left.\boldsymbol{\omega}^{\prime} \boldsymbol{\imath}\right)
\end{array}\right) \exp \left(r_{f} \hat{T}\right)+\sum_{i=1}^{N} \omega_{i} \exp \left(r_{f} \hat{T}+R_{i, T+\hat{T}}\right),
$$

where $R_{i, T+\hat{T}}$ denotes the cumulative excess $\log$ return between $T$ and $T+\hat{T}$, i.e., $R_{i, T+\hat{T}}=r_{i, T+1}+r_{i, T+2}+\ldots+r_{i, T+\hat{T}}$, and $\boldsymbol{l}$ is a vector of ones.

We assume that investor preferences can be represented by a utility function with constant relative risk aversion: $u(W)=\frac{W^{1} A}{1 A}$, with $A>1$. The problem can then be formally stated as follows:

$$
\begin{aligned}
& \max _{\ln } E_{T}\left\{\frac{\left[\left(\begin{array}{ll}
1 & \left.\omega^{\prime} \iota\right) \exp \left(r_{f} \hat{T}\right)+\sum_{i 1}^{N} \omega_{i} \exp \left(r_{f} \hat{T}+R_{i, T+\hat{T}}\right)
\end{array}\right]^{1 A}\right.}{1 A}\right\} \\
& \left\{\omega_{i}\right\}_{i 1}^{N} .
\end{aligned}
$$

We also assume the presence of short sale and borrowing restrictions, so portfolio weights are constrained between zero and one.

Finally, excess returns are generated by the VAR model:

$$
z_{t}=a+B x_{t} 1+\epsilon_{t}
$$

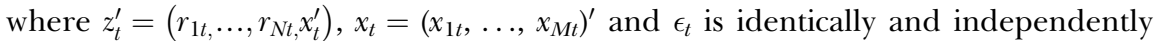
distributed according to $N(0, \Sigma)$. Vector $x_{t}$ is the vector of $M$ variables that predict expected returns. It is useful to rewrite the model as:

$$
z_{t}=a+B_{0} z_{t} 1+\epsilon_{t}
$$

where:

$$
B_{0}=\left[\mathbf{0}_{(N+M) \times N} B\right] .
$$

The sum $Z_{T+\hat{T}}=z_{T+1}+z_{T+2}+\ldots+z_{T+\hat{T}}$ is distributed as a multivariate normal with mean $\mu_{\text {sum }}$ and variance $\Sigma_{\text {sum }}$ given by:

$$
\begin{aligned}
\mu_{\text {sum }}= & \hat{T} a+\left(\begin{array}{ll}
\hat{T} & 1
\end{array}\right) B_{0} a+\left(\begin{array}{ll}
\hat{T} & 2
\end{array}\right) B_{0}^{2} a+\cdots+B_{0}^{\hat{T}}{ }^{1} a \\
& +\left(B_{0}+B_{0}^{2}+\cdots+B_{0}^{\hat{T}}\right) z_{T},
\end{aligned}
$$




$$
\begin{aligned}
\sum_{\text {sum }}= & \sum \\
& +\left(I+B_{0}\right) \Sigma\left(I+B_{0}\right)^{\prime} \\
& +\left(I+B_{0}+B_{0}^{2}\right) \sum\left(I+B_{0}+B_{0}^{2}\right)^{\prime} \\
& \vdots \\
& +\left(I+B_{0}+\cdots+B_{0}^{\hat{T}} 1\right) \Sigma\left(I+B_{0}+\cdots+B_{0}^{\hat{T}} \quad 1\right)^{\prime},
\end{aligned}
$$

Since a solution to (2) is not known in closed form, Barberis (2000) proposes to evaluate the objective function numerically by computing the integral:

$$
\int u\left(W_{T+\hat{T}}\right) p\left(\mathbf{R}_{T+\hat{T}} \mid \Phi_{T}\right) d \mathbf{R}_{T+\hat{T}},
$$

where $\mathbf{R}_{T+\hat{T}}$ is the column vector of cumulative excess $\log$ returns, $p\left(\mathbf{R}_{T+\hat{T}} / \Phi_{\mathrm{T}}\right)$ is the probability density function of the predictive distribution, and $\Phi_{T}$ is the investor's information set at $T$. The approach therefore involves estimating the parameters in (3) and then drawing realizations of $Z_{T+\hat{T}}$ from the multivariate normal distribution with mean and variance $\hat{\mu}_{\text {sum }}$ and $\hat{\Sigma}_{\text {sum. }}$. Next, for every simulated realization, the value of the integral's argument is calculated, and finally the arithmetic mean of all values is obtained. The numerical solution to the optimization problem is the portfolio choice that maximizes the integral's numerical value.

Barberis (2000) also considers the possibility that the investor decides to take estimation risk into account. The predictive distribution then results from integrating the joint distribution of future returns and the parameter vector in (3), denoted by $\theta$. Namely, the investor will obtain the probability density function for returns as:

$$
\begin{aligned}
p\left(\mathbf{R}_{T+\hat{T}} \mid \Phi_{T}\right) \equiv p\left(\mathbf{R}_{T+\hat{T}} \mid z\right) & =\int p\left(\mathbf{R}_{T+\hat{T}}, \theta \mid z\right) d \theta \\
& =\int p\left(\mathbf{R}_{T+\hat{T}} \mid \theta, z\right) p(\theta \mid z) d \theta,
\end{aligned}
$$

where $z=z_{1}, z_{2}, \ldots, z_{T}$. The probability density $p\left(\mathbf{R}_{T+\hat{t}} \theta, z\right)$ corresponds to a multi variate normal distribution with mean and variance equal to $\mu_{\text {sum }}$ and $\Sigma_{\text {sum }}$. An investor who takes parameter uncertainty into account therefore evaluates the following integral:

$$
\int u\left(W_{T+\hat{T}}\right) p\left(\mathbf{R}_{T+\hat{T}} \mid \theta, z\right) p(\theta \mid z) d \theta d \mathbf{R}_{T+\hat{T}} .
$$

In practical terms, a realization of the vector $\theta$ is first drawn from the posterior distribu tion, ${ }^{4} p(\theta \mid z)$, and then a possible realization of $\mathbf{R}_{T+\hat{T}}$ is simulated using the distribution of future returns conditional on those parameter values. In the empirical application of Section 3, our choices for the size of the simulated sample are 100,000 in the case of no parameter uncertainty and 10,000 when estimation risk is taken into account. 
If parameter uncertainty is ignored, it is possible to obtain an analytical approxi mation to the investor's objective function. Such closed form solution, albeit inexact, enables us to investigate the investment horizon effects documented by Barberis (2000) and Boudry and Gray (2003).

Since $A>1$, we can restate (2) as:

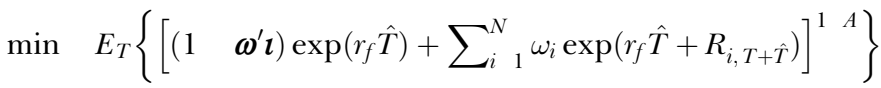

$$
\begin{aligned}
& \left\{\omega_{i}\right\}_{i \quad 1}^{N} \text {. }
\end{aligned}
$$

and, since wealth is always positive due to portfolio constraints, we can also write:

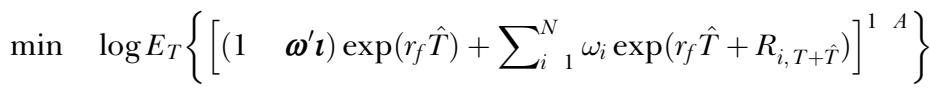

$$
\begin{aligned}
& \left\{\omega_{i}\right\}_{i}^{N} \text {. }
\end{aligned}
$$

Consider the following second order Taylor approximation for log of wealth adapted from Campbell and Viceira (2002):

$$
\begin{aligned}
& \log \left[\left(\begin{array}{ll}
1 & \boldsymbol{\omega}^{\prime} \boldsymbol{\imath}
\end{array}\right) \exp \left(r_{f} \hat{T}\right)+\sum_{i}^{N} \omega_{i} \exp \left(r_{f} \hat{T}+R_{i, T+\hat{T}}\right)\right] \\
& \approx r_{f} \hat{T}+\boldsymbol{\omega}^{\prime} \mathbf{R}_{T+\hat{T}}+\frac{1}{2} \boldsymbol{\omega}^{\prime} \boldsymbol{v}_{\hat{T}}^{2} \quad \frac{1}{2} \boldsymbol{\omega}^{\prime} V_{\hat{T}} \boldsymbol{\omega}^{\prime},
\end{aligned}
$$

where $\boldsymbol{v}_{\hat{T}}^{2}$ is a vector containing the variances of the elements in $\mathbf{R}_{T+\hat{T}}$, and $V_{\hat{T}}$ is the variance covariance matrix of $\mathbf{R}_{T+\hat{T}}$.

The approximation makes wealth lognormal and therefore enables us to write the objective function in mean variance terms:

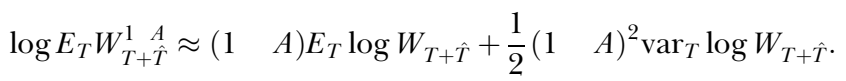

Dividing by (1 A) the objective function becomes:

$$
r_{f} \hat{T}+\boldsymbol{\omega}^{\prime}\left(E_{T} \mathbf{R}_{T+\hat{T}}+\frac{1}{2} \boldsymbol{v}_{\hat{T}}^{2}\right) \quad \frac{A}{2} \boldsymbol{\omega}^{\prime} V_{\hat{T}} \boldsymbol{\omega}^{\prime} .
$$

This expression has the traditional mean variance form except that: (i) excess returns are replaced by log excess returns when computing both the mean and the variance covariance terms, and (ii) mean log excess return of the $i$ th asset must be corrected by adding the term $\frac{1}{2} \nu_{i \hat{T}}^{2}=\frac{1}{2} \operatorname{var}_{T}\left(R_{i, T+\hat{T}}\right)$. For example, in the case of two risky assets, the investor maximizes:

$$
\begin{gathered}
r_{f} \hat{T}+\omega_{1}\left(E_{T} R_{1, T+\hat{T}}+\frac{1}{2} \operatorname{var}_{T}\left(R_{1, T+\hat{T}}\right)\right)+\omega_{2}\left(E_{T} R_{2, T+\hat{T}}+\frac{1}{2} \operatorname{var}_{T}\left(R_{2, T+\hat{T}}\right)\right) \\
\frac{A}{2}\left(\omega_{1}^{2} \operatorname{var}_{T}\left(R_{1, T+\hat{T}}\right)+\omega_{2}^{2} \operatorname{var}_{T}\left(R_{2, T+\hat{T}}\right)+2 \omega_{1} \omega_{2} \operatorname{cov}_{T}\left(R_{1, T+\hat{T}}, R_{2, T+\hat{T}}\right)\right)
\end{gathered}
$$

subject to $0 \leq \omega_{i} \leq 1, i=1,2$. 
In the particular case of a single risky investable asset, if $R_{T+\hat{T}}$ denotes the risky asset's cumulative log excess return, and $\omega$ the fraction of initial wealth invested in the risky asset, then the optimal portfolio is:

$$
\begin{array}{rlrl}
\omega^{*} & =0 & & \text { for } E_{T} R_{T+\hat{T}}+\frac{1}{2} \operatorname{var}_{T}\left(R_{T+\hat{T}}\right)<0, \\
\omega^{*}=1 & \text { for } E_{T} R_{T+\hat{T}}+\frac{1}{2} \operatorname{var}_{T}\left(R_{T+\hat{T}}\right)>A \operatorname{var}_{T}\left(R_{T+\hat{T}}\right) \\
\omega^{*}=\frac{E_{T} R_{T+\hat{T}}}{A \operatorname{var}_{T}\left(R_{T+\hat{T}}\right)}+\frac{1}{2 A} & \text { otherwise, }
\end{array}
$$

since the objective function is globally concave in $\omega$.

\section{(ii) Investment Horizon Effects}

In order to understand how the investment horizon changes the moments of cumulative log excess returns, we start by restricting our attention to the two risky asset case with multiple predictors, and then focus on the single risky asset, single predictor special case.

Assume that the two risky assets are a portfolio of stocks and a portfolio of bonds, denoted with subscripts $s$ and $b$, respectively. For the sake of tractability, we assume also that variables predicting stock and bond returns follow univariate first order autoregressive processes, although we allow for correlation in the error terms across variables. Return dynamics are therefore governed by the following VAR model:

$$
\begin{aligned}
& r_{s t+1}=\alpha_{s}+\beta_{s 1} x_{1 t}+\cdots+\beta_{s M} x_{M t}+\varepsilon_{s t+1} \\
& r_{b t+1}=\alpha_{b}+\beta_{b 1} x_{1 t}+\cdots+\beta_{b M} x_{M t}+\varepsilon_{b t+1} \\
& x_{1 t+1}=\gamma_{1}+\phi_{1} x_{1 t}+\varepsilon_{1 t+1} \\
& x_{M t+1}=\gamma_{M}+\phi_{M} x_{M t}+\varepsilon_{M t+1} \\
& \left(\begin{array}{c}
\varepsilon_{s t+1} \\
\varepsilon_{b t+1} \\
\varepsilon_{1 t+1} \\
\vdots \\
\varepsilon_{M t+1}
\end{array}\right) \sim N\left[0,\left(\begin{array}{ccccc}
\sigma_{s}^{2} & \sigma_{s b} & \sigma_{s 1} & \cdots & \sigma_{s M} \\
& \sigma_{b}^{2} & \sigma_{b 1} & \cdots & \sigma_{b M} \\
& & \sigma_{1}^{2} & & \sigma_{1 M} \\
& & & \ddots & \vdots \\
& & & & \sigma_{M}^{2}
\end{array}\right)\right]
\end{aligned}
$$

where we assume that $\left|\phi_{k}\right|<1, \forall k$. Let us study stock returns, since conclusions are identical for either risky asset. The first order stationary autocovariance of excess returns is given by:

$$
\operatorname{cov}\left(r_{s t}, r_{s t+1}\right)=\sum_{k 1}^{M} \beta_{s k} \sigma_{s k}+\sum_{k 1}^{M} \beta_{s k} \phi_{k} \sum_{j 1}^{M} \beta_{s j} \phi_{j} \frac{\sigma_{k j}}{1 \phi_{k} \phi_{j}} .
$$


If the predictive coefficient, $\beta_{s k}$, and the covariance between returns and the shock to the predictive variable, $\sigma_{s k}$, have opposite signs, then shocks to the pre dictive variable have opposite effects on the current period's return (through the contemporaneous covariance) and next period's expected return (through the predictive equation) contributing to negative serial correlation in stock returns. Similarly, negative values for $\beta_{s k} \beta_{s j} \phi_{k} \phi_{j} \sigma_{k j}$ tend to induce negative autocorrelation in the return series. Assume, for instance, that $\beta_{s k}, \beta_{s j}, \phi_{k}, \phi_{j}>0$ and $\sigma_{k j}<0$. In this case, a positive shock to $x_{k t}$ increases next period's expected return but is likely to be contemporaneous with a negative shock to $x_{j t}$, which, in turn, decreases $x_{j t+1}$ and therefore the expected value of $r_{s t+2}$. Negative serial correlation in returns, how ever, does not guarantee that the relative risk return trade off for the risky asset improves with the investment horizon. To see this, we derive, next, the expressions for the moments of cumulative returns.

From (5) and (8) it follows that, under stationarity, the conditional variance of $R_{s, T+\hat{T}}$ evolves according to:

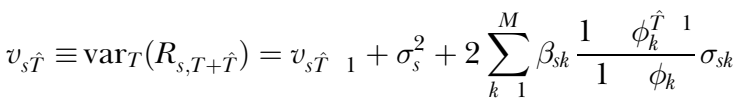

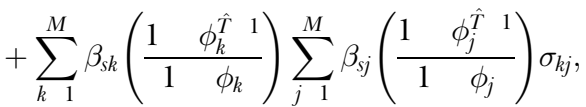

for $\hat{T}=1,2, \ldots, \infty$, with $v_{s 0}=0$.

Similarly, the cumulative mean of stock returns is given by:

$$
m_{s \hat{T}} \equiv E_{T}\left(R_{s, T+\hat{T}}\right)=m_{s \hat{T} \quad 1}+\alpha_{s}+\sum_{k 1}^{M} \beta_{s k}\left(\gamma_{k} \frac{1 \frac{\phi_{k}^{\hat{T}}}{1}{ }^{1} \phi_{k}}{1}+\phi_{k}^{\hat{T}}{ }^{1} x_{k T}\right)
$$

for $\hat{T}=1,2, \ldots, \infty$, with $m_{s 0}=0$.

To complete the analysis, the covariance of cumulative stock and bond returns evolves with $\hat{T}$ according to:

$$
\begin{aligned}
& \operatorname{cov}_{\hat{T}} \equiv \operatorname{cov}_{T}\left(R_{s, T+\hat{T}}, R_{b, T+\hat{T}}\right) \\
& =\operatorname{cov}_{\hat{T} 1}+\sigma_{s b}+\sum_{k 1}^{M} \beta_{s k} \frac{1 \phi_{k}^{\hat{T}} 1}{1} \phi_{k} \sigma_{s k}+\sum_{k}^{M} \beta_{b k} \frac{1}{1 \quad \phi_{k}^{\hat{T}} 1} \sigma_{b k}
\end{aligned}
$$

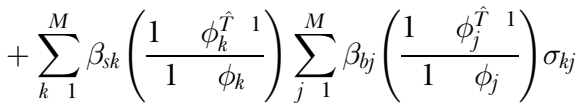

for $\hat{T}=1,2, \ldots, \infty$, with $\operatorname{cov}_{0}=0$.

Note that, if excess log returns were i.i.d. $\left(\beta_{i k}=0, i=s, b, \forall k\right)$, both the variance covariance matrix and the mean vector in (6) would be proportional to $\hat{T}$, so the investment horizon could be removed from the objective function and the optimal risky asset portfolio would have the same composition regardless of the investment horizon. In the non i.i.d. case, however, predictability in risky asset returns will cause the whole mean variance frontier to change with the investor's horizon, which 
will induce investment horizon effects both in the optimal allocation to the risky portfolio as well as in the composition of the risky portfolio itself.

More specifically, $v_{s} \hat{T}$ may grow more or less than linearly as the investor's horizon increases, depending on whether $v_{\hat{T}} \quad v_{\hat{T}} \quad$ is increasing in $\hat{T}$. For the simple case of two predictive variables $(M=2)$ and non negative $\phi_{1}, \phi_{2}$, changes in the conditional cumulative variance will decrease with the investment horizon if:

$$
\begin{aligned}
& \frac{\partial\left(v_{s \hat{T}} \quad v_{s \hat{T}} 1\right)}{\partial \hat{T}}=2 \beta_{s 1} \sigma_{s 1} \phi_{1}^{\hat{T}} 1 \frac{\ln \phi_{1}}{1+\phi_{1}}+2 \beta_{s 2} \sigma_{s 2} \phi_{2}^{\hat{T}} \quad 1 \frac{\ln \phi_{2}}{1+\phi_{2}}
\end{aligned}
$$

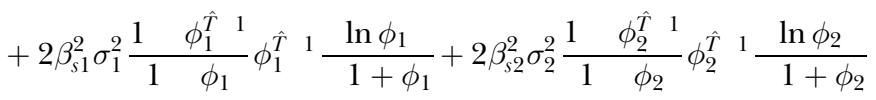

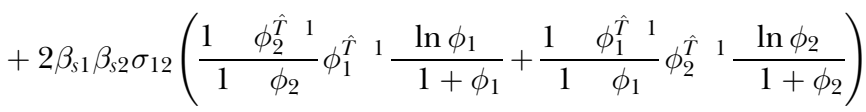

$$
\begin{aligned}
& <0 \text {. }
\end{aligned}
$$

It thus follows that for non negative $\phi_{k}$, if $\beta_{s k}$ and $\sigma_{s k}$, have opposite signs, the $k$ th predictive variable not only may induce negative serial correlation in the risky asset returns, but it also tends to reduce the relative riskiness of a long term investment in the risky asset as long as:

$$
\beta_{s k} \sigma_{s k}<\beta_{s k}^{2} \sigma_{k}^{2} \frac{1 \quad \phi_{k}^{\hat{T}} \quad 1}{1 \quad \phi_{k}}
$$

Also, when the effects of the predictive variables on the risky asset's expected return, i.e., $\beta_{s 1}$ and $\beta_{s 2}$, have the same (opposite) sign, and the contemporaneous covariance between both variables is positive (negative) the last term in the left hand side of (9) is negative, which contributes towards making the conditional variance of the risky asset's return grow less than linearly with the investor's horizon.

The cumulative mean, on the other hand, grows less than linearly with the investment horizon if:

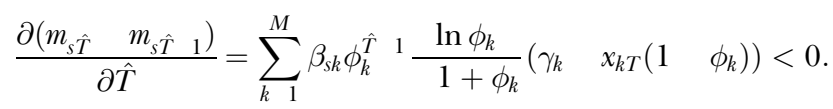

Boudry and Gray (2003) perform their comparative statics exercises by setting the initial value of one predictive variable below or above its stationary mean, $\frac{\gamma_{k}}{1 \phi_{k}}$, while leaving the rest of predictors initially at their long run means. It is clear from (10) that when the value of the perturbed variable is initially below (above) its stationary mean and $\beta_{s k}>0(<0)$, the mean of cumulative returns will grow more than linearly with the investment horizon.

Finally, the investment horizon also affects the composition of the risky asset portfolio as well as its total riskiness relative to that of the risk free asset through the covariance of risky asset returns. In particular, changes in the conditional 
covariance will decrease with the investment horizon if:

$$
\begin{aligned}
& \frac{\partial\left(\operatorname{cov}_{\hat{T}} \quad \operatorname{cov}_{\hat{T}} 1\right)}{\partial \hat{T}}=\beta_{s 1} \sigma_{s 1} \phi_{1}^{\hat{T}} 1 \frac{\ln \phi_{1}}{1+\phi_{1}}+\beta_{s 2} \sigma_{s 2} \phi_{2}^{\hat{T}} 1 \frac{\ln \phi_{2}}{1+\phi_{2}} \\
& +\beta_{b 1} \sigma_{b 1} \phi_{1}^{\hat{T}} 1 \frac{\ln \phi_{1}}{1+\phi_{1}}+\beta_{b 2} \sigma_{b 2} \phi_{2}^{\hat{T}} 1 \frac{\ln \phi_{2}}{1+\phi_{2}} \\
& +2 \beta_{s 1} \beta_{b 1} \sigma_{1}^{2} \frac{1}{1} \phi_{1}^{\hat{T}} \phi_{1} \phi_{1}^{\hat{T}} 1 \frac{\ln \phi_{1}}{1+\phi_{1}}+2 \beta_{s 2} \beta_{b 2} \sigma_{2}^{2} \frac{1 \quad \phi_{2}^{\hat{T}} 1}{1} \phi_{2} \phi_{2}^{\hat{T}} 1 \frac{\ln \phi_{2}}{1+\phi_{2}} \\
& +\left(\beta_{s 1} \beta_{b 2}+\beta_{s 2} \beta_{b 1}\right) \sigma_{12}\left(\frac{1}{1} \frac{\phi_{2}^{\hat{T}}}{1} \phi_{2} \phi_{1}^{\hat{T}} 1 \frac{\ln \phi_{1}}{1+\phi_{1}}+\frac{1}{1} \phi_{1}^{\hat{T}} \phi_{1} \phi_{2}^{\hat{T}} 1 \frac{\ln \phi_{2}}{1+\phi_{2}}\right)
\end{aligned}
$$

$<0$.

Clearly, under the stationarity assumption, in the long horizon limit $(\hat{T} \rightarrow \infty)$, the expressions in (9), (10) and (11) all converge to zero, which implies that the optimal portfolio becomes independent of the investment horizon for very large $\hat{T}$.

In order to reconcile the empirical results obtained by Barberis (2000) and Boudry and Gray (2003), we focus, next, on the single risky asset, single predictor case. It follows from (7) that positive horizon effects on the optimal unconstrained allocation to the risky asset are a consequence of the variance of cumulative log returns growing slower than mean cumulative log returns with $\hat{T}$.

The return generating model in the single risky asset, single predictor case becomes:

$$
\begin{aligned}
r_{t+1} & =\alpha+\beta x_{t}+\varepsilon_{s t+1} \\
x_{t+1} & =\gamma+\phi x_{t}+\varepsilon_{1 t+1} \\
\left(\begin{array}{c}
\varepsilon_{s t+1} \\
\varepsilon_{1 t+1}
\end{array}\right) & \sim N\left[0,\left(\begin{array}{cc}
\sigma_{s}^{2} & \sigma_{s 1} \\
\sigma_{s 1} & \sigma_{1}^{2}
\end{array}\right)\right] .
\end{aligned}
$$

Under the stationarity assumption, i.e., $|\phi|<1$, the first order autocovariance of excess returns is given by:

$$
\operatorname{cov}\left(r_{t}, r_{t+1}\right)=\beta \sigma_{s 1}+\frac{\beta^{2} \sigma_{1}^{2} \phi}{1 \phi^{2}}
$$

so returns are serially negatively correlated in the long run if $\beta \sigma_{s 1}<\beta^{2} \sigma_{1}^{2} \frac{\phi}{1 \frac{\phi^{2}}{2}}$.

The variance of the cumulative return between periods $T$ and $T+\hat{T}$ evolves with $\hat{T}$ according to:

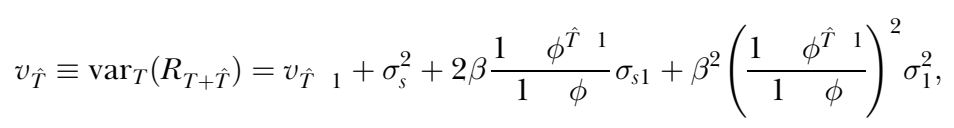

which holds for $\hat{T}=1,2, \ldots, \infty$, with $v_{0}=0$. For non negative $\phi$, one period changes in the variance of cumulative returns are decreasing in the horizon if: 


$$
\begin{aligned}
& \frac{\partial\left(v_{\hat{T}} \quad v_{\hat{T}} 1\right)}{\partial \hat{T}}=2 \beta \sigma_{s 1} \phi^{\hat{T}} 1 \frac{\ln \phi}{1+\phi}+2 \beta^{2} \sigma_{1}^{2} \frac{1}{1 \quad \phi} \phi^{\hat{T}} 11 \phi^{\hat{T}} 1 \frac{\ln \phi}{1+\phi} \\
& <0
\end{aligned}
$$

so the variance grows less than linearly in $\hat{T}$, for $1<\hat{T}<\infty$, if the following condi tion holds:

$$
\beta \sigma_{s 1}<\beta^{2} \sigma_{1}^{2} \frac{1 \phi^{\hat{T}} 1}{1 \phi} .
$$

Note that, even if $\beta$ and $\sigma_{s 1}$ have opposite signs, it is not possible to assert whether their joint effect is enough to cause the variance to always grow less than linearly. With negatively serially correlated returns, $v_{\hat{T}}$ could be concave in $\hat{T}$ at short hor izons but convex at longer horizons.

As for the mean of cumulative returns:

$$
m_{\hat{T}} \equiv E_{T}\left(R_{T+\hat{T}}\right)=m_{\hat{T} 1}+\alpha+\beta \gamma \frac{1 \phi^{\hat{T}}{ }^{1}}{1 \quad \phi}+\beta \phi^{\hat{T}}{ }^{1} x_{T}
$$

for $\hat{T}=1,2, \ldots, \infty$, with $m_{0}=0$. The mean increases in $\hat{T}$ only if $\alpha+\beta \gamma \frac{1 \phi^{T} 1}{1 \phi}+\beta \phi^{\hat{T}}{ }^{1} x_{T}>0$. So, even if the variance of cumulative returns grows less than linearly in the investment horizon, a diminishing mean might cause the optimal allocation to decrease for long horizons. Moreover, assuming that the mean is positive and increasing in $\hat{T}$, it could grow slower than the variance. To see this, note that differentiating $m_{\hat{T}} \quad m_{\hat{T}}$ 1 with respect to $\hat{T}$ gives:

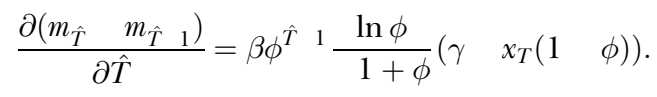

So, for $\hat{T}<\infty$, the mean of cumulative returns $m_{\hat{T}}$ grows less than linearly with $\hat{T}$ if the predictor's initial value is above (below) its long run mean when $\beta$ is positive (negative), and more than linearly otherwise. Note that the effect of the predictor's initial value on $\frac{\partial\left(m_{T} m_{T}{ }_{1}\right)}{\partial \hat{T}}$ has an opposite sign to that on $m_{\hat{T}}$. As a consequence, when $x_{T}$ affects $E_{T}\left(r_{T+\hat{T}}\right)$ positively, its increments decrease as the horizon becomes longer if the predictor is initially above its long run mean. Intuitively, when the predictive variable is stationary, the influence of the predictor's initial value on each period's return decays with time. The larger the initial effect of the predicting variable, the larger the loss in the cumulative expected return as $\hat{T}$ increases.

Finally, under stationarity, the unconstrained optimal allocation to the risky asset converges to a constant:

$$
\omega^{*}=\frac{\alpha+\frac{\beta \gamma}{1} \phi}{A\left(\sigma_{s}^{2}+\frac{2 \beta \sigma s_{1}}{1 \phi}+\left(\frac{\beta}{1 \phi}\right)^{2} \sigma_{1}^{2}\right)}+\frac{1}{2 A} .
$$


Convergence, however, is far from obvious in Barberis's (2000) results for US stocks because of the high persistence in the predictor $(\phi=0.9774)$, but becomes evident in Boudry and Gray (2003), where $\phi$ ranges from 0.9039 to 0.9191 .

To conclude this section, note that if returns are unpredictable, i.e. $\beta=0$, then $v_{\hat{T}}=\hat{T} \sigma_{1}^{2}$ and $m_{\hat{T}}=\hat{T} \alpha$, and the unconstrained optimal allocation becomes:

$$
\omega^{*}=\frac{\alpha}{A \sigma_{s}^{2}}+\frac{1}{2 A}
$$

regardless of the investment horizon.

\section{EMPIRICAL RESULTS}

In this section, we analyze empirically the effect of the investor's time horizon on his optimal asset allocation. We start by considering the case when only a single risky asset is available for investment and only one variable has predictive power over the risky asset return, and then extend the analysis to the more general model with two risky assets and multiple predictors.

As the single risky asset we consider, alternatively, a long term US Government bond portfolio and a portfolio of US stocks, whose returns are proxied by the return on a representative constant maturity portfolio of 10 year US Government Bonds and on the value weighted index of the New York Stock Exchange, respectively. Data were collected with monthly frequency for the period covering January 1959 to December 1998 from the CRSP (Center for Research in Security Prices) database. In order to compute excess returns, we also employ the one month Treasury Bill rate series. When the constant risk free rate is needed, we take $r_{f}$ to be 0.0036 , or $4.32 \%$ in annual terms.

Two predictive variables are selected for predicting stock and bond returns, respectively: the dividend yield on the stock index (DP), computed as the sum of dividends payed on the index over the previous 12 months divided by the current index level; and the trend of the stock index (Trend), defined as the difference between the logarithm of the index's current level and the average level over the previous 12 months.

We consider first the case of an investor who estimates the parameters in (12) and takes as the predictive distribution of returns a multivariate normal distribution with mean and variance $\hat{\mu}_{\text {sum }}$ and $\hat{\Sigma}_{\text {sum }}$, respectively. Values of the OLS parameter estimates in both cases are shown in Table 1. Figure 1 shows graphically the optimal allocation to stocks. The first conclusion we draw from Figure 1 is that the approxi mate solution performs remarkably well in this specific setting. Second, the investor allocates a larger fraction of his wealth to stocks the higher the value of DP and the longer the investment horizon. This result is consistent with Brennan, Schwartz and Lagnado (1997), Barberis (2000) or Kritzman and Rich (1998). In fact, it is to be expected from the results of the previous section for two reasons. First, condition (15) together with estimated parameters implies that the variance of cumulative returns grows less than linearly for any finite investment horizon. Second, the high estimated value of $\phi$, close to unity, prevents conditional expected returns from reverting fast to the mean. As a consequence, the horizon effect on the variance 
Table 1

Parameter Values in the Model with a Single Risky Asset and One Predictor

\begin{tabular}{lcc}
\hline Panel A & Constant & DP \\
\hline Stocks & 0.0044 & 0.0025 \\
DP & 0.0477 & 0.9862 \\
\hline & Constant & Trend \\
\hline 10 year Bonds & 0.0025 & 0.0007 \\
Trend & 0.2121 & 0.8787 \\
\hline Panel B & & \\
\hline Stocks & Stocks & DP \\
DP & 0.0019 & 0.9296 \\
\hline & & 0.0290 \\
\hline 10 year Bonds & 10 year Bonds & Trend \\
Trend & 0.0005 & 0.2690 \\
\hline
\end{tabular}

Notes:

This table shows OLS estimates from the VAR model below. Panel A displays regression coefficients while Panel B shows the variance covariance matrix of the error terms. $r_{t}$ denotes the continuously compounded monthly excess return on the risky asset (alternatively, US stocks or 10 year US Government bonds) and $x_{t}$ is the single predictor: the dividend yield (DP) or the trend of the stock index (Trend). In Panel B, values above the main diagonal are correlation coefficients.

$$
\begin{array}{cc}
r_{t+1} & \alpha+\beta x_{t}+\varepsilon_{1 t+1} \\
x_{t+1} & \gamma+\phi x_{t}+\varepsilon_{2 t+1} \\
\left(\begin{array}{c}
\varepsilon_{1 t+1} \\
\varepsilon_{2 t+1}
\end{array}\right) \sim N\left[0,\left(\begin{array}{cc}
\sigma_{s}^{2} & \sigma_{s 1} \\
\sigma_{s 1} & \sigma_{1}^{2}
\end{array}\right)\right]
\end{array}
$$

dominates that on the mean, and the optimal allocation to stocks is increasing for all investment horizons and all initial values of $x$ considered.

The case of long term bonds is quite different even though the unconditional autocovariance of excess returns is also negative. In Figure 2 we plot the investor's optimal allocation to long term US Government bonds for different investment horizons and three different cases that correspond to three different initial values of Trend. A pattern very similar to that documented by Boudry and Gray (2003) is found: since the coefficient in the regression equation for Trend is negative, the optimal demand is smaller but increasing in the investment horizon for an initial value above the predictor's sample mean, and larger but decreasing for a lower initial value. In particular, an investor with a relative risk aversion coefficient of 5 doubles his allocation to bonds when his investment horizon increases from 2.5 years to 10 years if the trend's initial value is high. These results are therefore consistent with mean driven horizon effects dominating variance driven effects. 


\section{Figure 1}

\section{Optimal Allocation to Stocks}

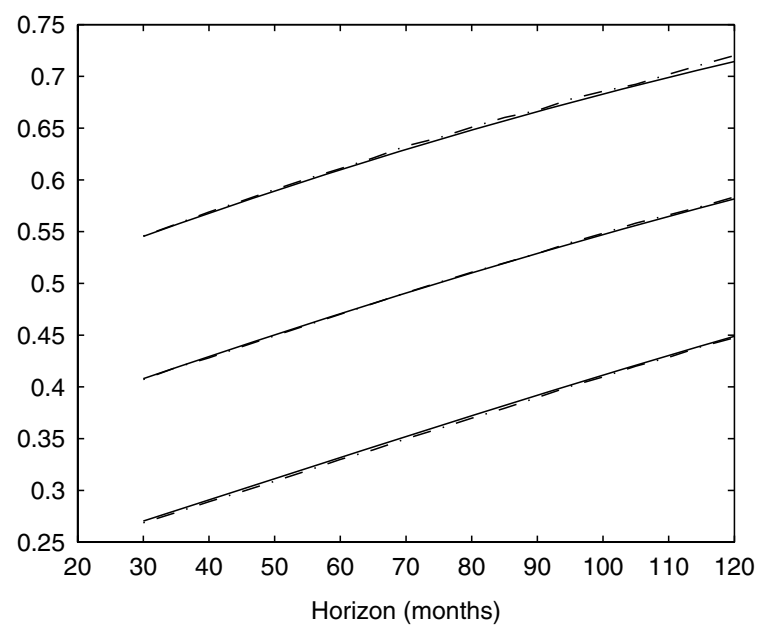

Notes:

This figure shows the optimal allocation to stocks as a function of the investment horizon when dividend yield (DP) is the only predictor, using the approximate analytical solution (solid lines) and the exact numerical solution (dash dot lines). The bottom line corresponds to an initial value of DP equal to 3 , the middle line to 4 , and the top line to 5 . The risk aversion coefficient is 10 .

Next, we solve for the investor's portfolio choice when both risky assets are avail able for investment. In this case, we include two additional predictive variables: ${ }^{5}$

1. The default spread, proxied by the yield difference between Moody's Baa and Aaa rated corporate bonds.

2. The term spread, proxied by the yield difference between 10 year and one year US Government Bonds.

Both series were obtained from the Internet site maintained by the US Federal Reserve.

If the investor performed an OLS regression of monthly excess stock and bond returns on the predictive variables in the previous month, he would obtain the following estimated coefficients and their associated $p$ values (in parentheses):

$$
\begin{aligned}
& r_{s t+1}=0.0077+0.0066 x_{1 t}+0.0007 x_{2 t} \\
& \begin{array}{lll}
(0.2181) & (0.1608) \quad(0.4257)
\end{array} \\
& +0.0054 x_{3 t} \quad 0.0004 x_{4 t}+\epsilon_{s t+1} \\
& (0.0071) \quad(0.2291)
\end{aligned}
$$




\section{Figure 2}

Optimal Allocation to Long term US Government Bonds

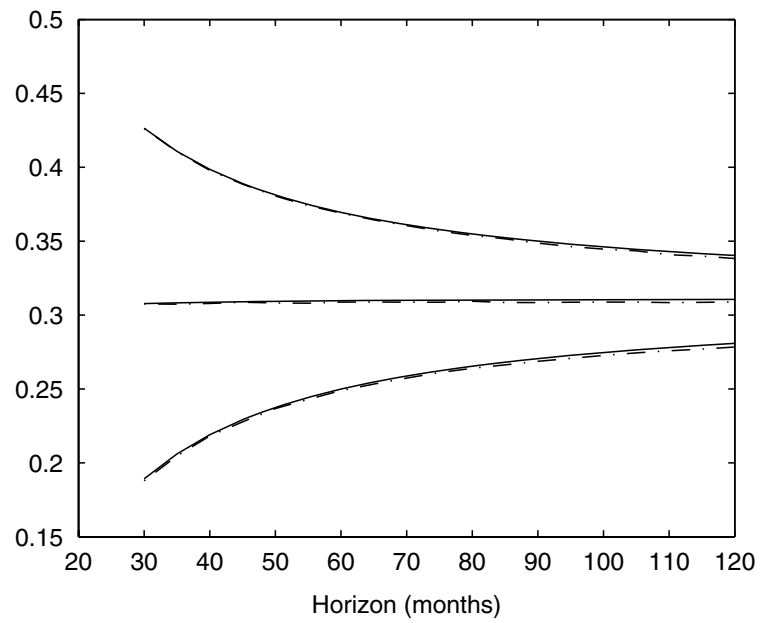

Notes:

This figure shows the optimal allocation to long term US Government bonds as a function of the investment horizon when the trend of the stock index (Trend) is the only predictor, using the approxi mate analytical solution (solid lines) and the exact numerical solution (dash dot lines). The top line corresponds to an initial value of Trend equal to 1 , the middle line corresponds to 2 , and the bottom line to 5 . The risk aversion coefficient is 10 .

$$
\begin{aligned}
& r_{b t+1}=0.0002+0.0038 x_{1 t} 0.0008 x_{2 t} \\
& (0.4814)(0.1333) \quad(0.3288) \\
& +0.0024 x_{3 t} \quad 0.0009 x_{4 t}+\epsilon_{b t+1} \\
& (0.0140) \quad(0.0010)
\end{aligned}
$$

where $r_{s t}$ and $r_{b t}$ stand for continuously compounded excess returns on the stock and the 10 year bond indices respectively, and $x_{i t}, i=1,2,3$, and 4 denote the value in month $t$ of the default spread, DP, the term spread, and the stock index trend, respectively.

In regression (18) only the term spread is significantly different from zero, whereas in regression (19) the term spread and especially the trend have a signifi cant predictive power over next month's expected return. On the other hand, the $R^{2}$ coefficient equals 0.0211 in the case of stocks and 0.0319 for bonds. These results show the magnitude of the investor's problem of deciding whether he should consider the empirical evidence on predictability or whether he should think that evidence is too weak to take the regression estimated coefficients as the true model parameter values. The Bayesian approach can thus be seen as an intermediate solution between both extreme attitudes. Next, we solve for the optimal portfolio choice of an investor who ignores estimation risk and an investor who explicitly 
Table 2

Parameter Values in the Model with Two Risky Assets and Four Predictors

\begin{tabular}{|c|c|c|c|c|c|c|}
\hline Panel A & \multicolumn{2}{|c|}{ Constant } & Def. Spread & $D P$ & Term Spread & Trend \\
\hline Stocks & 0.0 & & 0.0066 & 0.0007 & 0.0053 & 0.0004 \\
\hline 10 year Bonds & 0.0 & & 0.0038 & 0.0008 & 0.0024 & 0.0009 \\
\hline Def. spread & 0.0 & & 0.9499 & 0.0208 & 0.0142 & 0.0047 \\
\hline DP & 0.0 & & 0.0462 & 1.0014 & 0.0195 & 0.0022 \\
\hline Term spread & 0.0 & & 0.1522 & 0.0416 & 0.9581 & 0.0126 \\
\hline Trend & 0.5 & & 0.3902 & 0.0791 & 0.1989 & 0.8705 \\
\hline \multirow[t]{2}{*}{ Panel B } & & & & & & \\
\hline & Stocks & Bonds & Def. Spread & $D P$ & Term Spread & Trend \\
\hline Stocks & 0.0019 & 0.2695 & 0.0783 & 0.9379 & 0.1399 & 0.9903 \\
\hline Bonds & & 0.0005 & 0.2485 & 0.2827 & 0.1620 & 0.2624 \\
\hline Def. spread & & & 0.0102 & 0.0990 & 0.1634 & 0.0723 \\
\hline DP & & & & 0.0289 & 0.1935 & 0.9325 \\
\hline Term spread & & & & & 0.0704 & 0.1318 \\
\hline Trend & & & & & & 3.5845 \\
\hline
\end{tabular}

Notes:

When the investor does not take parameter uncertainty into account, he takes the parameter values in the VAR model below as known. More specifically, parameter values in this table are the means from the parameter posterior distribution under a diffuse prior. Panel A displays regression coefficients while Panel B shows the variance covariance matrix of the error terms. $r_{s t}$ denotes the continuously com pounded monthly excess return on US stocks, $r_{b t}$ denotes the continuously compounded monthly excess return on 10 year US Government bonds, $x_{1 t}, x_{2 t}, x_{3 t}, x_{4 t}$, are the default spread, the dividend yield (DP), the term spread, and the trend of the stock index (Trend). In Panel B, values above the main diagonal are correlation coefficients.

$$
\begin{aligned}
& r_{s t+1} \quad \alpha_{s}+\beta_{s 1} x_{1 t}+\beta_{s 2} x_{2 t}+\beta_{s 3} x_{3 t}+\beta_{s 4} x_{4 t}+\varepsilon_{s t+1} \\
& r_{b t+1} \quad \alpha_{b}+\beta_{b 1} x_{1 t}+\beta_{b 2} x_{2 t}+\beta_{b 3} x_{3 t}+\beta_{b 4} x_{4 t}+\varepsilon_{b t+1} \\
& x_{1 t+1} \quad \gamma_{1}+\phi_{11} x_{1 t}+\phi_{12} x_{2 t}+\phi_{13} x_{3 t}+\phi_{14} x_{4 t}+\varepsilon_{1 t+1} \\
& x_{2 t+1} \quad \gamma_{2}+\phi_{21} x_{1 t}+\phi_{22} x_{2 t}+\phi_{23} x_{3 t}+\phi_{24} x_{4 t}+\varepsilon_{2 t+1} \\
& x_{3 t+1} \quad \gamma_{3}+\phi_{31} x_{1 t}+\phi_{32} x_{2 t}+\phi_{33} x_{3 t}+\phi_{34} x_{4 t}+\varepsilon_{3 t+1} \\
& x_{4 t+1} \quad \gamma_{4}+\phi_{41} x_{1 t}+\phi_{42} x_{2 t}+\phi_{43} x_{3 t}+\phi_{44} x_{4 t}+\varepsilon_{4 t+1} \\
& \left(\begin{array}{c}
\varepsilon_{s t+1} \\
\varepsilon_{b t+1} \\
\varepsilon_{1 t+1} \\
\varepsilon_{2 t+1} \\
\varepsilon_{3 t+1} \\
\varepsilon_{4 t+1}
\end{array}\right) \sim N\left[0,\left[\begin{array}{cccccc}
\sigma_{s}^{2} & \sigma_{s b} & \sigma_{s 1} & \sigma_{s 2} & \sigma_{s 3} & \sigma_{s 4} \\
& \sigma_{b}^{2} & \sigma_{b 1} & \sigma_{b 2} & \sigma_{b 3} & \sigma_{b 4} \\
& & \sigma_{1}^{2} & \sigma_{12} & \sigma_{13} & \sigma_{14} \\
& & & \sigma_{2}^{2} & \sigma_{23} & \sigma_{24} \\
& & & & \sigma_{3}^{2} & \sigma_{34} \\
& & & & & \sigma_{4}^{2}
\end{array}\right)\right]
\end{aligned}
$$

incorporates estimation risk in his portfolio decision problem. When the investor does not recognize estimation risk, it will be assumed that he takes as the true parameter values the means of model parameters from the posterior distribution under a diffuse prior. These values are shown in Table 2.

Figure 3 summarizes the results graphically when all predictors' starting values coincide with their sample means. In the case of no parameter uncertainty, a long 


\section{Figure 3}

Optimal Portfolio Choice as a Function of the Investment Horizon
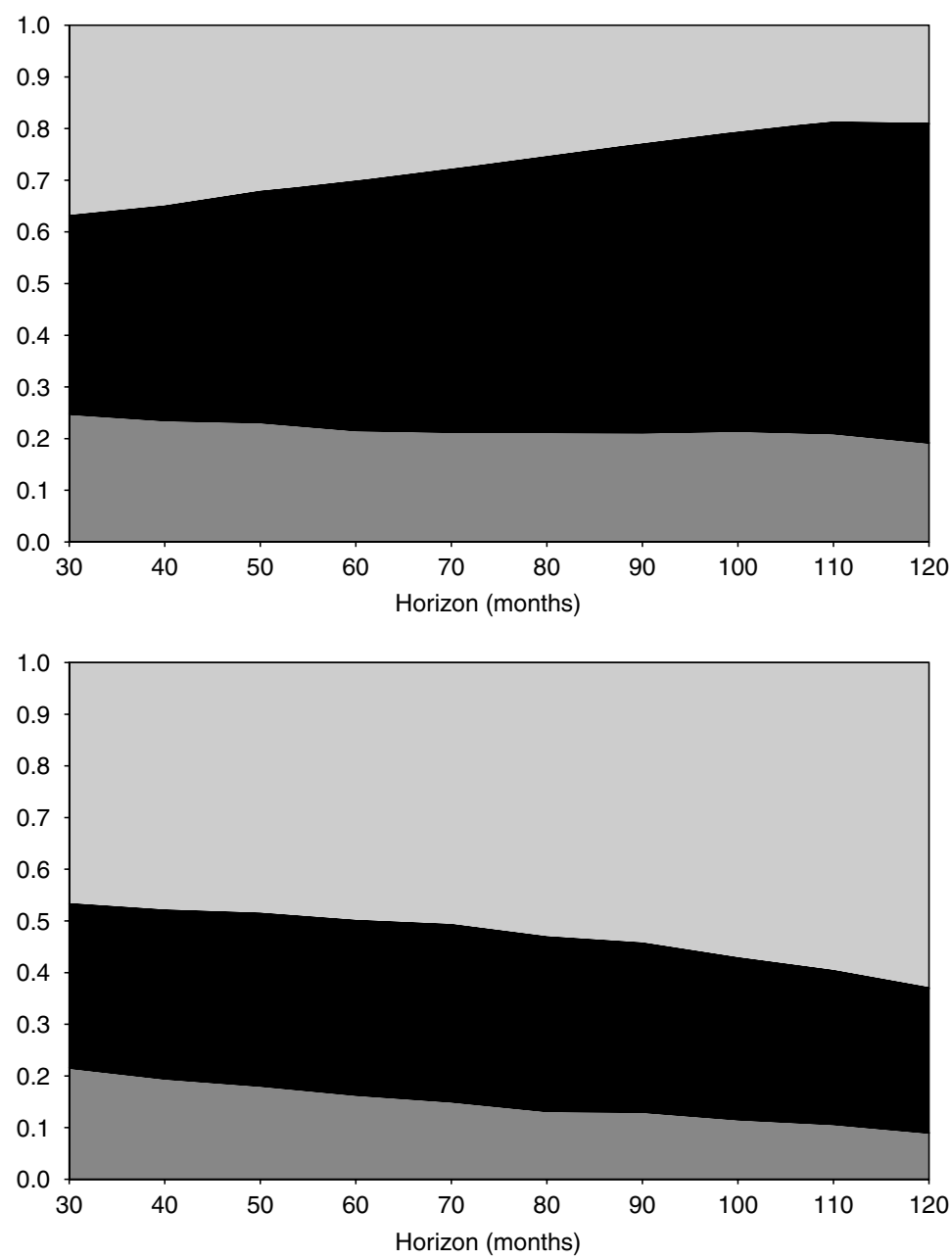

Notes:

The graph plots the static allocation to the riskless asset (light shade), 10 year bonds (medium shade) and stocks (dark shade) when returns are assumed to be generated by a VAR model with four predictors. The initial values of all predictive variables are set at their sample means. In the lower graph, the investor takes parameter uncertainty into account. The risk aversion coefficient is 10 .

term investor will invest a higher fraction of his risky asset portfolio in stocks and, therefore, a lower fraction in bonds than a short term investor. This consequence of predictability is interesting because the optimal allocation to bonds is indepen dent of the investor's horizon when bonds are the only risky investable asset. Clearly, the sharp decline in the relative riskiness of equity as the investment 
horizon increases tilts the optimal risky portfolio towards a smaller investment in the other risky asset: long term bonds. As a consequence, long term bonds account for almost $40 \%$ of a 30 month investor's risky portfolio, and only $23 \%$ in the case of a 10 year investor. This substitution effect, however, is almost completely compen sated by a larger overall investment in the risky asset portfolio, whose attractiveness relative to the safe investment increases with the investment horizon: allocation to bonds declines by only $5 \%$ of the total portfolio as the horizon grows from 30 to 120 months. When estimation risk is considered, the decline in the relative riskiness of stocks is offset by the larger effect of parameter uncertainty at long horizons, so the optimal allocation to stocks remains almost constant. Long term bonds, however, become relatively more risky for long investment horizons so the long term investor replaces bonds with the risk free asset.

Note that investment horizon effects in Figure 3 are solely driven by changes in the variance covariance matrix, since the horizon is likely to have a negligible effect on the conditional mean vector according to (10). In Figures 4 to 7 we set the initial value of one of the predictors above or below its sample mean.

Graphs in Figure 4 correspond to an initial value of Trend equal to its 25th sample percentile for the cases of parameter certainty and parameter uncertainty. Changes in the trend's initial value affect expected returns on stocks and bonds in the same direction: a low current value of the variable raises next period's conditional mean returns on both assets. Consequently, a short term investor allocates more to the risky asset portfolio than in the previous case. As the investment horizon increases, however, expected single period returns decline due to mean reversion. As a con sequence, the risk return trade off deteriorates for long term bonds while stocks become a more interesting investment alternative due to their lower relative risk, so the weight of bonds in the optimal risky portfolio sharply decreases from $46 \%$ to $26 \%$, while total investment in the risky portfolio increases due to the stronger appeal of stocks. Estimation risk, however, severely penalizes the long term investor's position in risky assets, and induces a negative horizon effect on the optimal alloca tion both to bonds and stocks. This finding is consistent both with Barberis (2000) and Boudry and Gray (2003).

Figure 5 shows the results corresponding to the case when Trend's initial value is set at its 75th sample percentile. Although the predictor's effect on expected bond returns suggests that long term bonds are a better investment at long horizons, the optimal fraction of bonds in the risky asset portfolio remains almost the same at both short and long horizons. Given the improvement in the risky investment opportunity set at long horizons, investment in the risk free asset reduces from $60 \%$ to $23 \%$ as $\hat{T}$ increases from 30 to 120 months. In this case, larger estimation risk at long horizons is also offset by the better risk return trade off, so the optimal portfolio has a similar composition both at long and short horizons when parameter uncertainty is taken into account.

It is interesting to note that although Figure 2 shows that the consequences of changes in the initial value of Trend on the investment horizon effect are symmetric around its mean, the presence of stocks in the investment opportunity set reinforces the negative horizon effect (allocation to bonds drops by almost 16\% in the horizons considered) and mitigates the positive horizon effect (allocation to bonds increases by $8 \%$ ).

Finally, we consider changes in the dividend yield's initial value. This predictor is particularly interesting because its autocorrelation coefficient is close to 1 according 


\section{Figure 4}

Optimal Portfolio Choice as a Function of the Investment Horizon
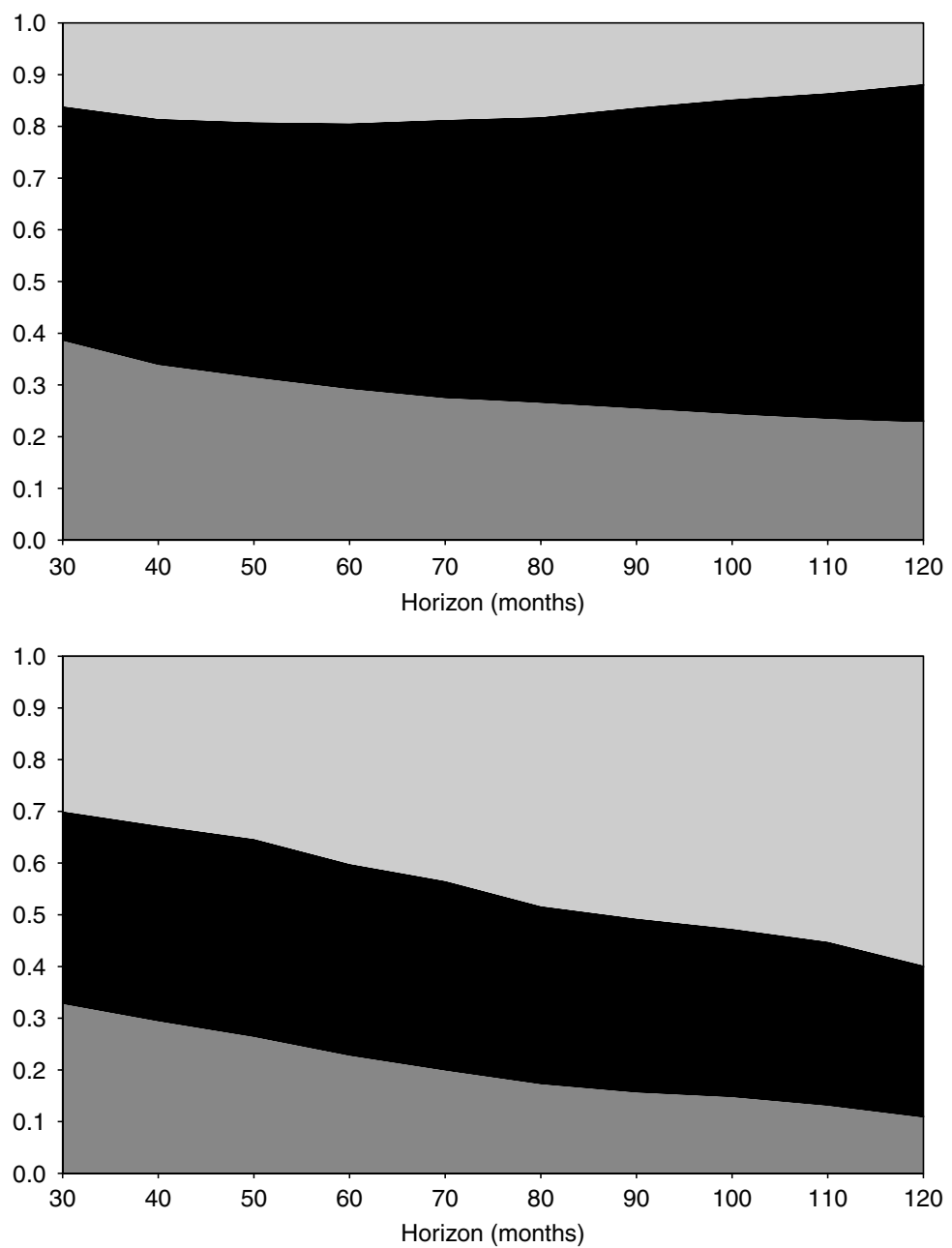

Notes:

The graph plots the static allocation to the riskless asset (light shade), 10 year bonds (medium shade) and stocks (dark shade) when returns are assumed to be generated by a VAR model with four predictors. The current value of predictor Trend (the trend of the stock index) equals 0.7 , while the initial values of the rest of predictive variables are set at their sample means. In the lower graph, the investor takes parameter uncertainty into account. The risk aversion coefficient is 10 .

to Table 2. This suggests that the investment horizon effects on the mean of cumulative returns are negligible due to lack of mean reversion in the predictor. Investment horizon effects in the optimal allocation to bonds can therefore only be 


\section{Figure 5}

Optimal Portfolio Choice as a Function of the Investment Horizon
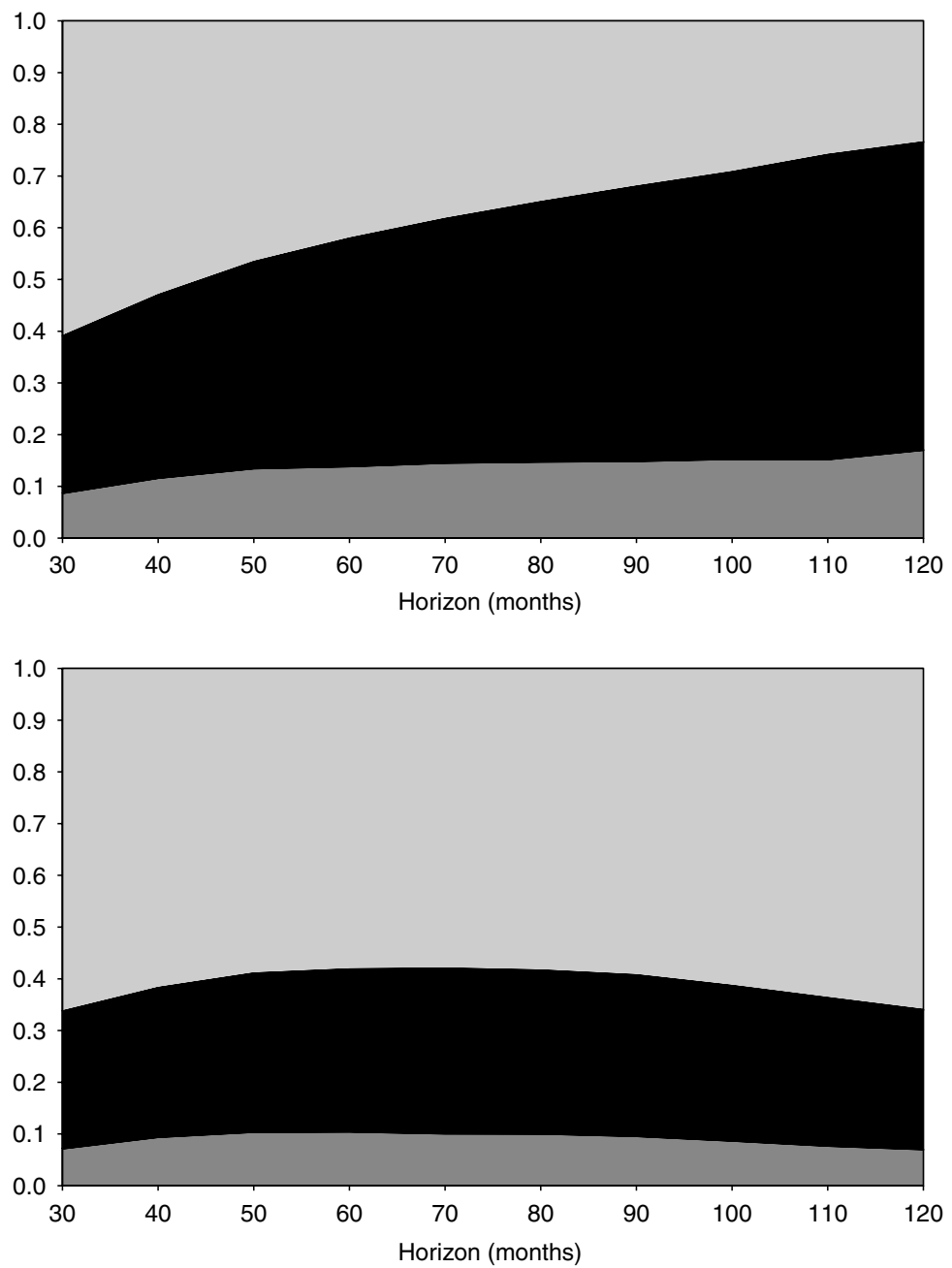

Notes:

The graph plots the static allocation to the riskless asset (light shade), 10 year bonds (medium shade) and stocks (dark shade) when returns are assumed to be generated by a VAR model with four predictors. The current value of predictor Trend (the trend of the stock index) equals 4.5 , while the initial values of the rest of predictive variables are set at their sample means. In the lower graph, the investor takes parameter uncertainty into account. The risk aversion coefficient is 10 .

negative and explained by either the lower riskiness of stocks as the horizon increases or by parameter uncertainty.

Figure 6 corresponds to the case when DP is initially at its 25 th sample percentile. As expected, the presence of long term bonds in the risky portfolio decreases with 


\section{Figure 6}

Optimal Portfolio Choice as a Function of the Investment Horizon
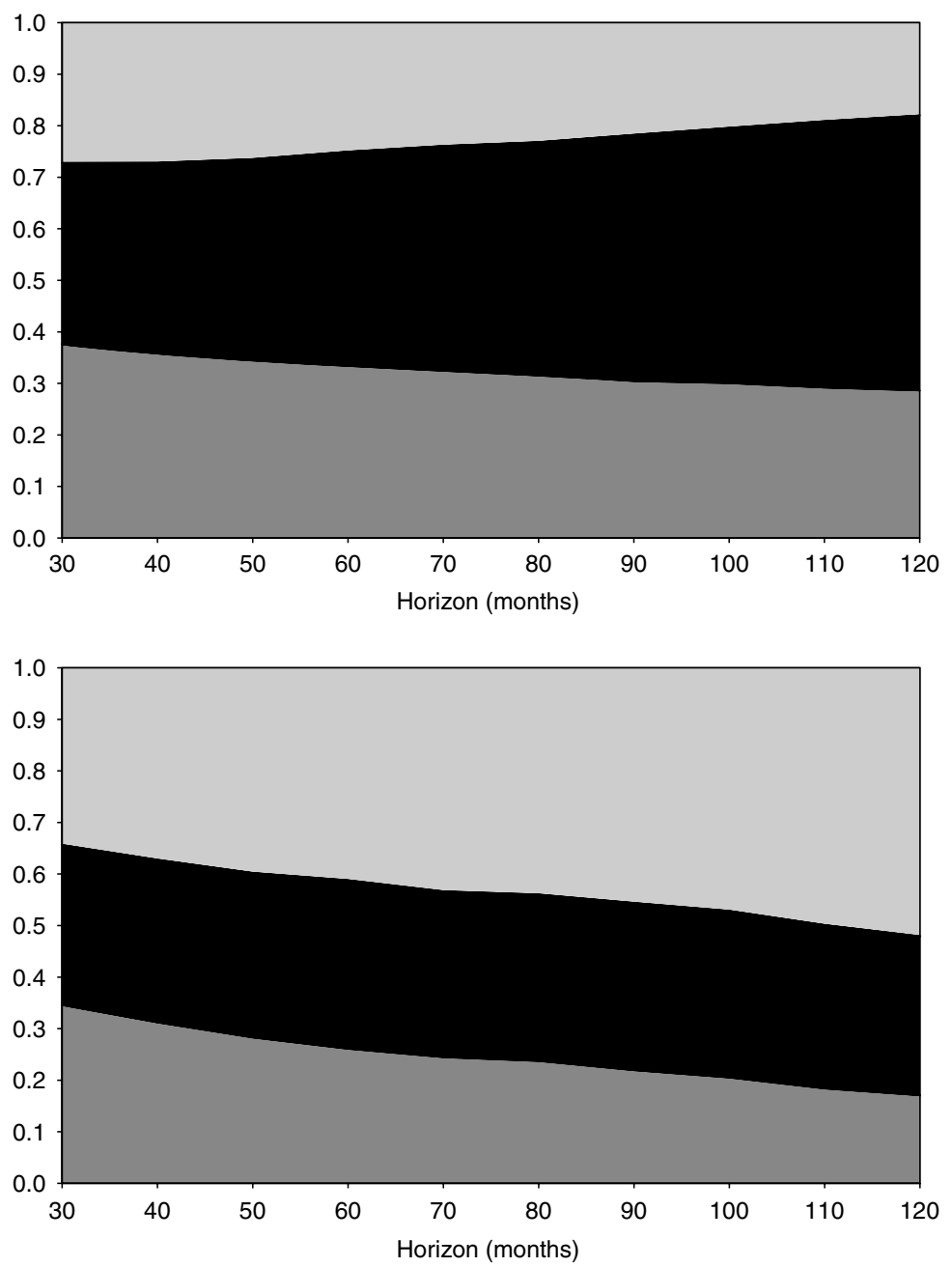

Notes:

The graph plots the static allocation to the riskless asset (light shade), 10 year bonds (medium shade) and stocks (dark shade) when returns are assumed to be generated by a VAR model with four predictors. The current value of predictor DP (dividend yield) equals 2.88, while the initial values of the rest of predictive variables are set at their sample means. In the lower graph, the investor takes parameter uncertainty into account. The risk aversion coefficient is 10 .

the investment horizon at the same rate as in Figure 3, where all predictors were set at their initial values. Because investment in the risky asset portfolio increases, horizon effects in the optimal allocation to bonds are negative but not very 


\section{Figure 7}

Optimal Portfolio Choice as a Function of the Investment Horizon
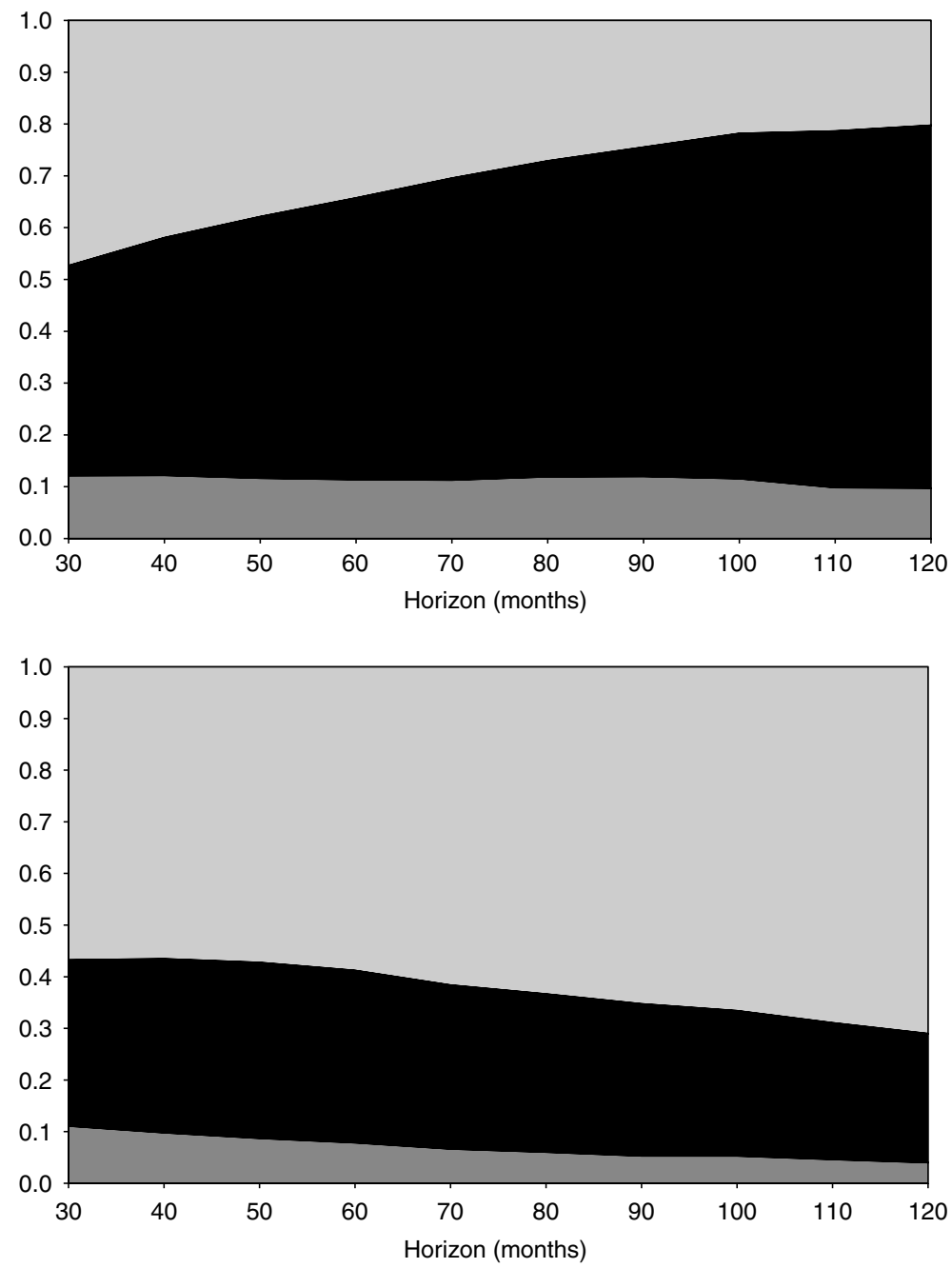

Notes:

The graph plots the static allocation to the riskless asset (light shade), 10 year bonds (medium shade) and stocks (dark shade) when returns are assumed to be generated by a VAR model with four predictors. The current value of predictor DP (dividend yield) equals 4.07, while the initial values of the rest of predictive variables are set at their sample means. In the lower graph, the investor takes parameter uncertainty into account. The risk aversion coefficient is 10 .

pronounced if the investor ignores estimation risk. Finally, Figure 7 shows the results for an initial value of DP equal to its 75 th sample percentile. Again, invest ment horizon effects on bond investment are negative. In this case, however, given 
the predominance of stocks in the optimal portfolio, changes in the investor's allocation to long term bonds are very small in relative terms, and only become apparent when parameter uncertainty is considered explicitly.

\section{CONCLUSIONS}

This paper takes a deeper look into the consequences of return predictability on investment horizon effects in the optimal buy and hold asset allocation of a utility maximizing investor.

The paper's first contribution is a theoretical analysis of how the parameters in the model driving the return dynamics influence the evolution of the investor's opportunity set with the investment horizon. It is shown that under stationarity of the predictive variables, all investment horizons disappear in the long horizon limit. When a single risky asset is available for investment, it is demonstrated that changes in the variance of cumulative returns are not necessarily monotonic in the invest ment horizon. It is also shown that the investment horizon can have either a positive or a negative effect on the mean of cumulative returns. This is due to a diminishing influence of the predictor's effect on expected returns when the state variable is stationary. If this effect is positive and the predictor's initial value is above average, a long term investor who believes in return predictability will expect a relatively lower return than a short term investor.

The paper also contributes to the literature on multi period asset allocation by extending Barberis (2000) and Boudry and Gray (2003) to the portfolio choice problem when two risky assets long term US Government Bonds and US stocks are available for investment both under parameter certainty and parameter uncer tainty. The main conclusion is that under return predictability the risk return characteristics of each risky asset can either improve or worsen with the investment horizon. The weight of each risky asset in the risky portfolio may therefore change as its relative appeal with respect to the other risky asset varies with the investment horizon.

\section{REFERENCES}

Barberis, N. (2000), 'Investing for the Long Run when Returns are Predictable', Journal of Finance, Vol. 55, No. 1 (February), pp. 22564.

Bodie, Z. (1995), 'On the Risk of Stocks in the Long Run', Financial Analysts Journal, Vol. 51, No. 3 (May/June), pp. 1822.

Boudry, W. and P. Gray (2003), 'Assessing the Economic Significance of Return Predictability: A Research Note', Journal of Business Finance E' Accounting, Vol. 30, Nos. 9\&10 (Nov./Dec.), pp. 130526.

Brennan, M.J., E.S. Schwartz and R. Lagnado (1997), 'Strategic Asset Allocation', Journal of Economic Dynamics and Control, Vol. 21, Nos. 8\&9 (June), pp. 1377403.

Campbell, J.Y. (1987), 'Stock Returns and the Term Structure', Journal of Financial Economics, Vol. 18, No. 2 (June), pp. 37399.

and R.J. Shiller (1988a), 'The Dividend Price Ratio and Expectations of Future Dividends and Discount Factors', Review of Financial Studies, Vol. 1, No. 3 (Fall), pp. 195228.

(1988b), 'Stock Prices, Earnings, and Expected Dividends', Journal of Finance, Vol. 43, No. 3 (July), pp. 66176 . 
Campbell J.Y. and L.M. Viceira (2002), Strategic Asset Allocation: Portfolio Choice for Long Term Investors, Clarendon Lectures in Economics (New York: Oxford University Press).

Connelly, T.J. (1996), 'The Time Diversification Controversy', Journal of Financial Planning (February), pp. 2023.

Fama, E.F. (1984), 'The Information in the Term Structure', Journal of Financial Economics, Vol. 13, No. 4 (December), pp. 50928.

(1990), 'Term Structure Forecasts of Interest Rates, Inflation, and Real Returns', Journal of Monetary Economics, Vol. 25, No. 1 (January), pp. 5976.

and K.R. French (1988), 'Dividend Yields and Expected Stocks Returns', Journal of Financial Economics, Vol. 22, No. 1 (October), pp. 325.

(1989), 'Business Conditions and Expected Returns on Stocks and Bonds', Journal of Financial Economics, Vol. 25, No. 1 (November), pp. 2349.

Keim, D.B. and R.F. Stambaugh (1986), 'Predicting Returns in the Stock and Bond Markets', Journal of Financial Economics, Vol. 17, No. 2 (December), pp. 35790.

Kritzman, M. (1994), 'What Practitioners Need to Know about Time Diversification', Financial Analysts Journal, Vol. 50, No. 1 (January/February), pp. 1418.

and D. Rich (1998), 'Beware of Dogma: The Truth About Time Diversification', Journal of Portfolio Management, Vol. 24, No. 4 (Summer), pp. 6677.

Merton, R. (1969), 'Lifetime Portfolio Selection Under Uncertainty: The Continuous Time Case', Review of Economics and Statistics, Vol. 51, No. 3 (August), pp. 24757.

(1971), 'Optimum Consumption and Portfolio Rules in a Continuous Time Model', Journal of Economic Theory, Vol. 3, No. 4 (December), pp. 373413.

Samuelson, P.A. (1963), 'Risk and Uncertainty: A Fallacy of Large Numbers', Scientia, Vol. 98 (April May), pp. 10813.

(1969), 'Lifetime Portfolio Selection by Dynamic Stochastic Programming', Review of Economics and Statistics, Vol. 51, No. 3 (August), pp. 23946.

Zellner, A. (1971), An Introduction to Bayesian Inference in Econometrics (New York: John Wiley \& Sons Ltd). 